\title{
The Effect of Material Surface Microstructure on the Enhancement of Bone-Bonding Ability of a Hydroxyapatite-Implant by Low-Intensity Pulsed Ultrasound (LIPUS)
}

\author{
Masanori Kobayashi \\ Department of Physical Therapy, College of Life and Health Sciences, Chubu University, Kasugai, Japan \\ Email: m-bear@rf7.so-net.ne.jp
}

How to cite this paper: Kobayashi, M. (2021) The Effect of Material Surface Microstructure on the Enhancement of BoneBonding Ability of a Hydroxyapatite-Implant by Low-Intensity Pulsed Ultrasound (LIPUS). Materials Sciences and Applications, 12, 42-51.

https://doi.org/10.4236/msa.2021.121003

Received: December 1, 2020

Accepted: January 17, 2021

Published: January 20, 2021

Copyright $\odot 2021$ by author(s) and Scientific Research Publishing Inc. This work is licensed under the Creative Commons Attribution International License (CC BY 4.0).

http://creativecommons.org/licenses/by/4.0/ (c) (i) Open Access

\begin{abstract}
Excellent firm bonding between the biomaterials and bone tissue (osseointegration and osteo-conductivity) has been desired for the stability in vivo of dental implants and artificial joints. Much has been learned about this concept, which has led to significant improvements in the design and surface modification of implants in the field of implant dentistry, orthopedic surgery. We have already reported that low-intensity pulsed ultrasound (LIPUS) irradiation can accelerate the bone bonding ability of the bio-conductive materials such as bioactive titanium and hydroxyapatite implant. However, it is still unclear whether the LIPUS could have same effect to different types of the bioactive-materials. Therefore, in this study, the differences of bone-like hydroxyapatite formation on some kind of hydroxyapatite surface in simulated body fluid (SBF) under the LIPUS irradiation were investigated. Two kinds of hydroxyapatite samples immersed in SBF was exposed to ultrasound waves, the bone-like apatite on the surface was analyzed by Scanning electron microscopy and X-ray diffraction. As a result, the enhancement of hydroxyapatite formation on the surface by LIPUS was confirmed, the initial epitaxial nucleation and crystal growth of apatite depended on crystal structure of the surface of matrix materials.
\end{abstract}

\section{Keywords}

Low-Intensity Pulsed Ultrasound (LIPUS), Simulated Body Fluid (SBF), Hydroxyapatite (HA), Osseointegration, Osteo-Conductivity

\section{Introduction}

The favorable clinical performance of current dental implants and artificial joints 
replacement have been attributed to their firm bonding through direct contact between living bone and the surface of the load-carrying implant at the microappearance level, which is often recognized as an osseointegration or osteo-conductivity [1] [2]. In particular, hydroxyapatite $\left(\mathrm{HA} ; \mathrm{Ca}_{10}\left(\mathrm{PO}_{4}\right)_{6}(\mathrm{OH})_{2}\right)$ has an excellent biocompatibility and bio-conductivity properties; therefore, it has become widely used as a coating material for dental implants and artificial joint [3] [4].

From a clinical viewpoint, more early bonding between a dental implant and the maxilla bone, or artificial joint stem and femoral bone will accelerate the onset of activity of patients sooner after an operation, with subsequent good long-term results. Therefore, approaches have been needed to accelerate osseointegration or bone bonding to HA material. Against this background, to enhance the direct attachment of implants with bone tissue, basic and clinical studies have tried [5] [6] [7].

We have already reported that low-intensity pulsed ultrasound (LIPUS) irradiation could accelerate bone-like hydroxyapatite precipitation on the bioactive titanium and hydroxyapatite surface, which results in firm bone bonding of implant [8] [9] [10]. This finding suggested the excellent attachment between the bio-active materials and bone tissue needs the achievement of bone-like crystalline formation on material surface, and that is not due to biological circumstances but the factor as physical phenomenon as a osseointegrarion mechanism. LIPUS therapy has been shown to enhance osteogenesis and is widely used as clinical treatment for complicated bone fractures in the field of orthopedic surgery, while, our study implied further potential as other clinical application of LIPUS. However, it is still unclear whether the ultrasound wave irradiation could have same effect to different types of the HA samples. Therefore, to get more information regarding clinical application for the enhancement of bone-implant attachment by the ultrasound wave irradiation, in this study, the differences of bone-like apatite formation on some kind of HA surface in simulated body fluid (SBF) under LIPUS irradiation was compared and investigated.

\section{Materials and Method}

As a basic bone-bonding ability test, a simulated body fluid (SBF) soaking method was performed in accordance with the method of Kokubo and colleagues in order to evaluate the apatite-forming ability of bio-active titanium and Bio-glass ceramics [11] [12] [13].

As an HA specimen, two kinds of commercial HA, CELLYARD pellet ${ }^{\circledR}$ (HOYA, Japan; 99.9\% HA: Sample-1 $\varnothing 5 \mathrm{~mm} \times 2 \mathrm{~mm}$ ) and NEOBONE- $\mathrm{X}^{\oplus}$ (Covalent Materials Co., Japan: Sample-2.) were used.

Hank's balanced solution (Lonza ${ }^{\oplus}$, USA) was used as an SBF, and maintained at over $\mathrm{pH} 7.0$ and $37^{\circ} \mathrm{C}$ and replaced every 2 days.

Ultrasound radiation was applied using a Sonic Accelerated Fracture Healing System (SAFHS; Smith \& Nephew, Memphis, TN, USA; Teijin Pharma, Tokyo, 
Japan). The treatment head module delivered ultrasound waves with $1.5 \mathrm{~Hz}$ sine waves, $200 \mu \mathrm{sec}$ signal term, and spatial average intensity of $30 \mathrm{~mW} / \mathrm{cm}^{2}$.

Each HA sample was soaked in SBF and subjected to ultrasound stimulation for 20 min daily for study periods of 3 days, 1 week, and 2 weeks. As a control, the same samples were left in SBF without ultrasound radiation in the same experimental conditions (Figure 1).

In order to compare with the effect of ultrasound waves, the HA surface was subjected to scanning electron microscopy (SEM) observation and X-ray diffraction (XRD) analyses, which focused on the top surfaces that received the direct radiation with ultrasound waves.

Furthermore, the each changes of sample mass were measured periodically. The value of the increased mass obtained dividing by the surface area was defined as the amount of precipitate per unit of surface area of the material. Statistical analysis of the data was performed using $\mathrm{t}$-test and the mean and standard deviation were calculated. Repeated measures ANOVA was used for comparisons between two groups, and the level of significance was determined to be at $\mathrm{p}<0.05$.

\section{Results}

\subsection{Scanning Electron Microscopy Observations}

\subsubsection{The Comparison of SEM Image between LIPUS Group and Control Group}

Figure 2 shows SEM images of the texture of the HA specimen surface in the LIPUS and control groups in the immersion experiments after 3 days, 1 week, and 2 weeks.

In both groups, morphological changes in the precipitations on the HA substrate surface are present according to the length of the soaking period.

As for the comparison of control group and LIPUS group, the crystallization changes on the surface demonstrated more active progress and growth in the LIPUS group in both cases of HA samples- 1 and 2 .

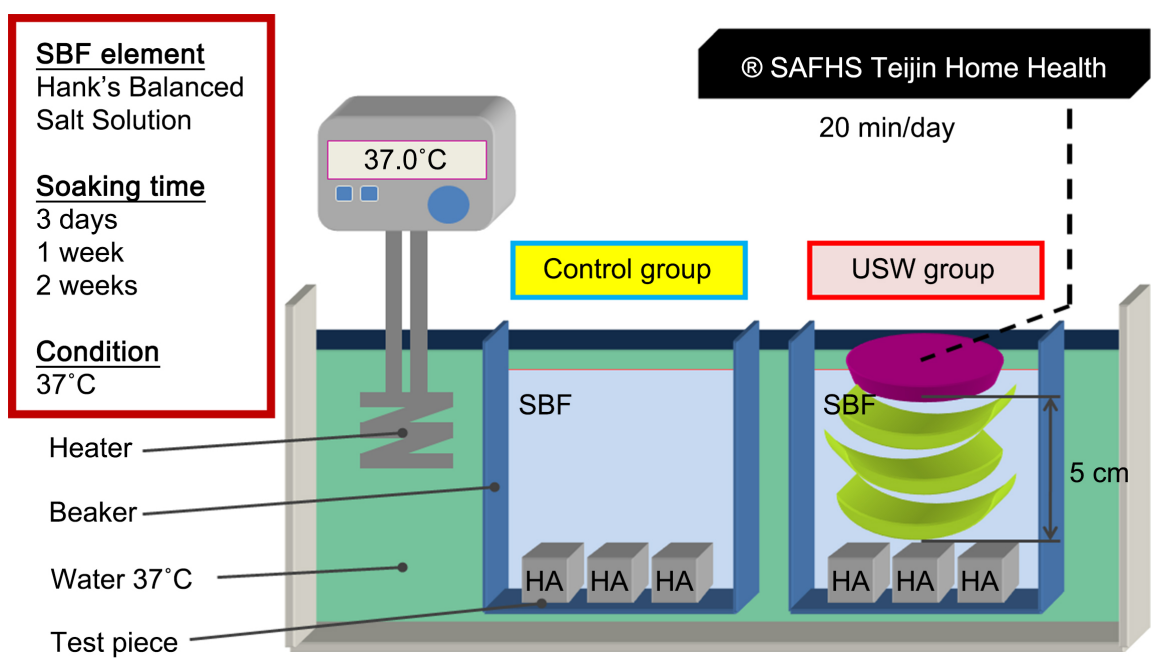

Figure 1. Schematic diagram of pulsed ultrasound wave radiation on HA specimens in SBF. 

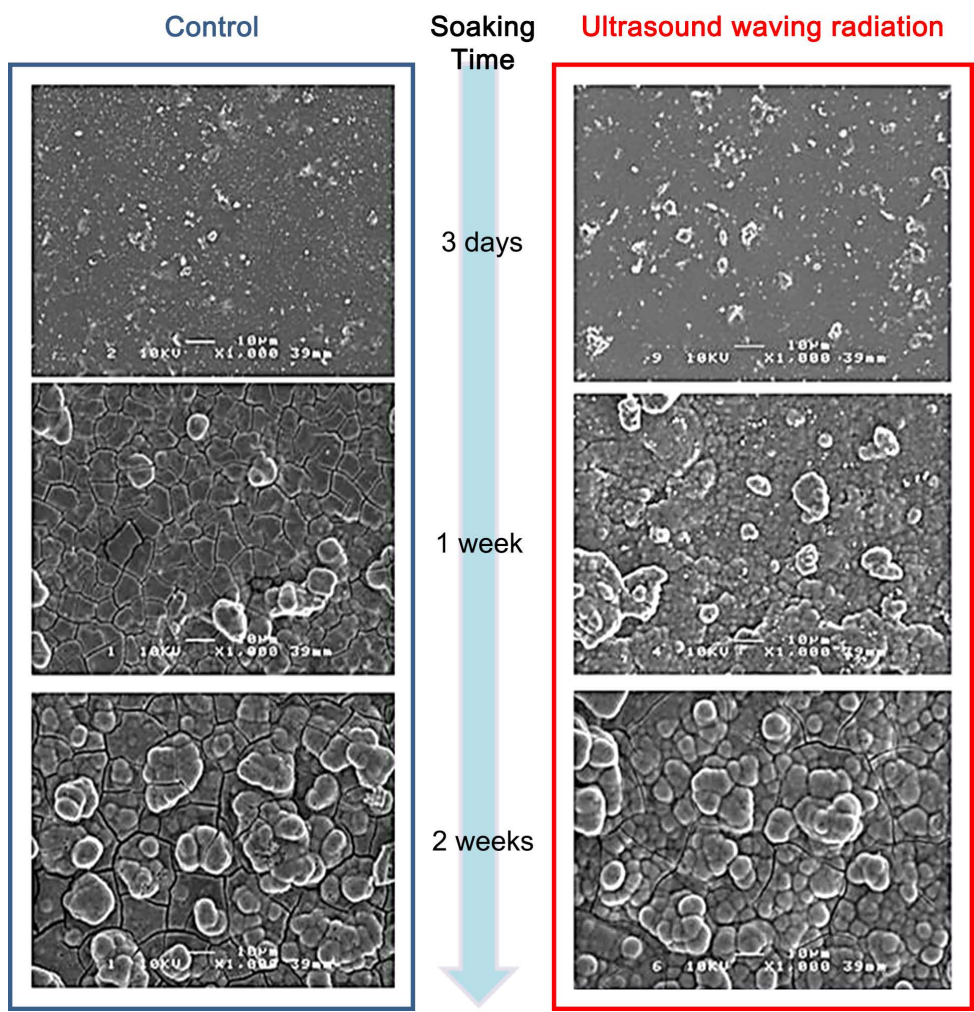

(a)
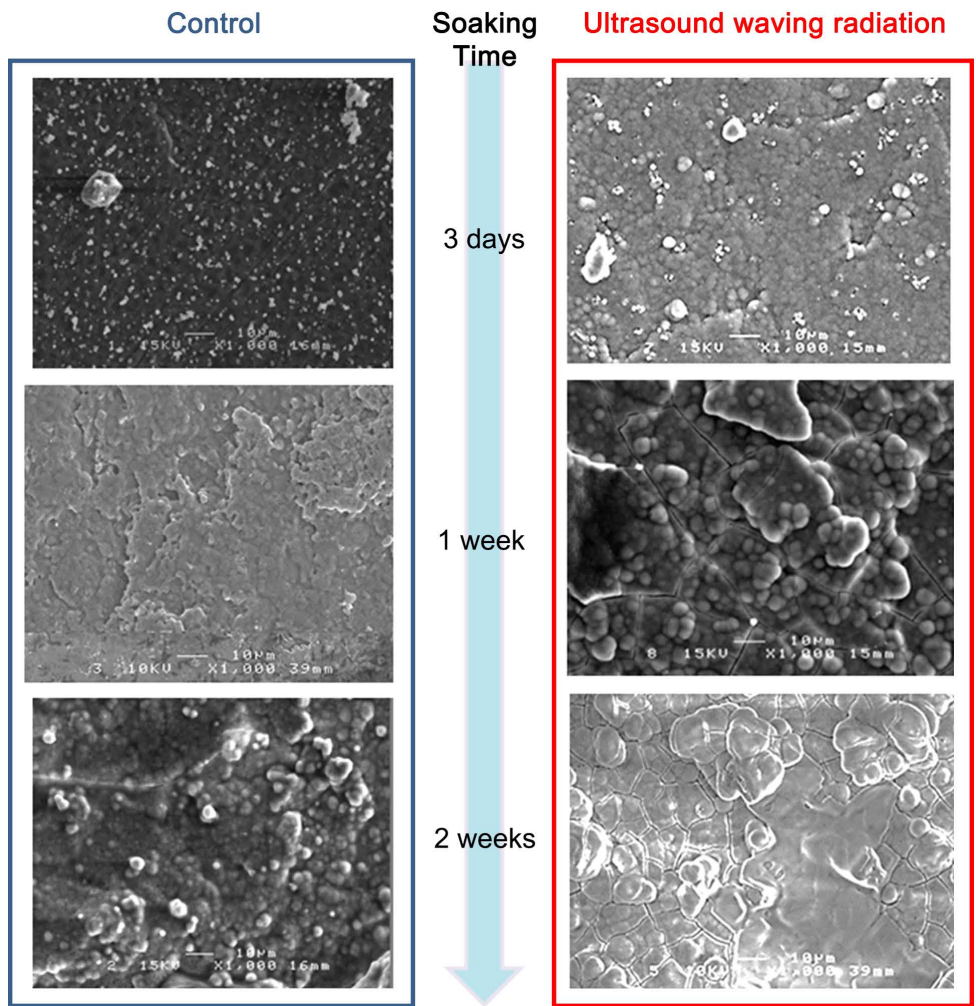

(b)

Figure 2. Scanning electron microscopy (SEM) micrographs of the surface of HA specimens after SBF soaking $(1000 \times)$. (a) Sample-1 (CELLYARD pellet $\left.^{\circledR}\right)$; (b) Sample-2 (NEOBONE-X ${ }^{\circledR}$. 
3.1.2. The Comparison of SEM Image between Two Kinds of HA Samples Figure 3 shows SEM images of the texture of two kinds of HA sample surfaces in the LIPUS after 3 days, 1 week, and 2 weeks in the SBF immersion.

Comparing sample- 1 and sample-2, the amount of precipitation in 3 days showed significant difference each other. Depending on the LIPUS irradiation time, the rapid crystallization of both samples had been succeed, finally covered the surface matrix with similar grain-like morphology. This suggested that LIPUS could be effective for acceleration of the crystal growth rather than initial nucleation.

\subsection{X-Ray Diffraction Analysis (XRD)}

The compounds on the HA surface were also identified by XRD. The XRD patterns of two kinds of HA samples before/after SBF soaking are shown in Figure 4.

Before soaking in SBF, the XRD patterns of both of samples indicated the hydroxyapatite structure, the reflection spectra of the sample-1 surface were focused on one point, while sample- 2 showed some peaks of hydroxyapatite. After soaking for 2 weeks, more discrete spectra of apatite were observed in sample- 2 . There were many identified other peaks in both specimens surface, they were speculated to be unfinished hydroxyapatite crystals under the crystallization.
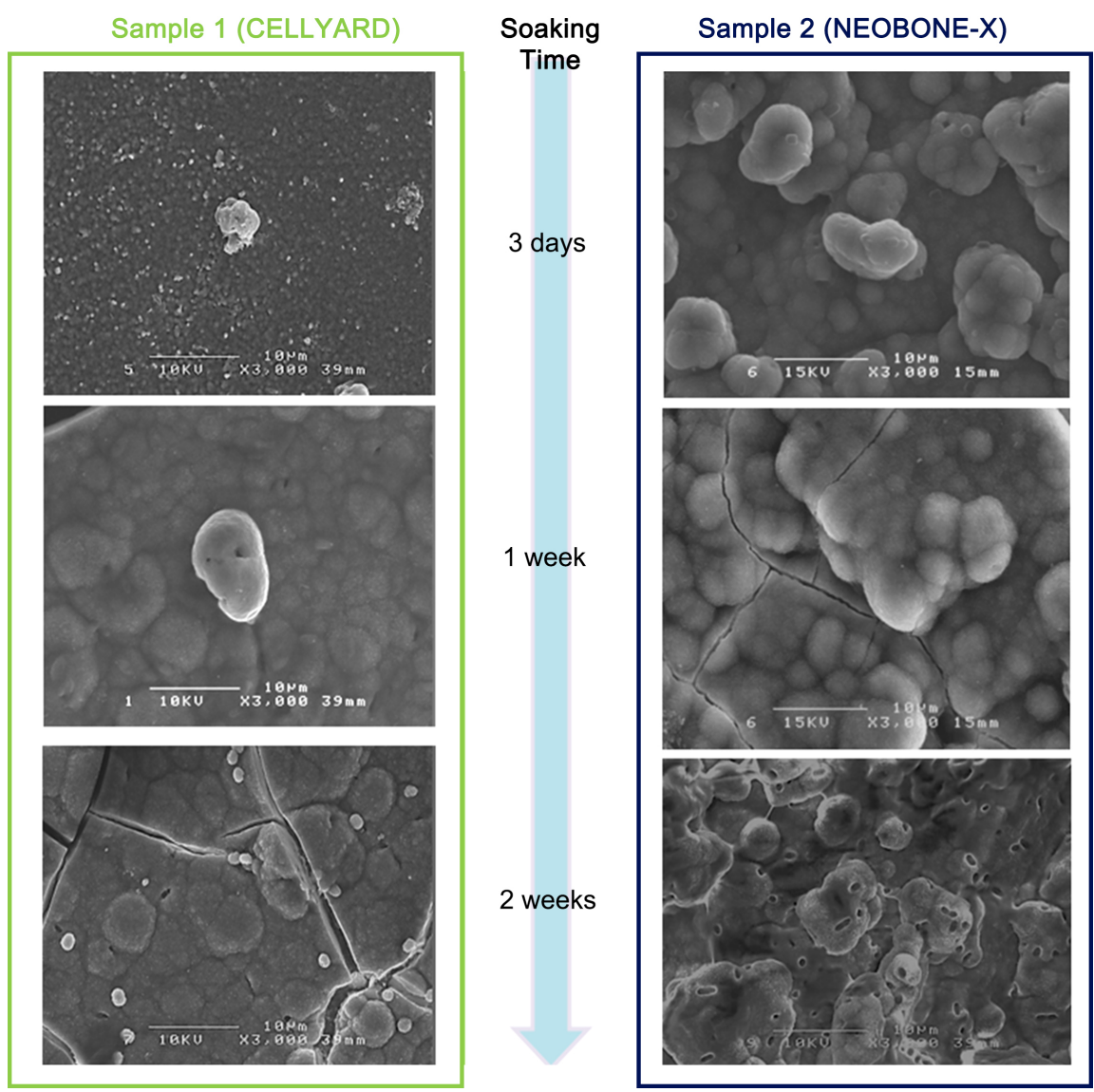

Figure 3. SEM micrographs of the surface of two kinds of HA samples after SBF soaking. $(1000 \times)$. 


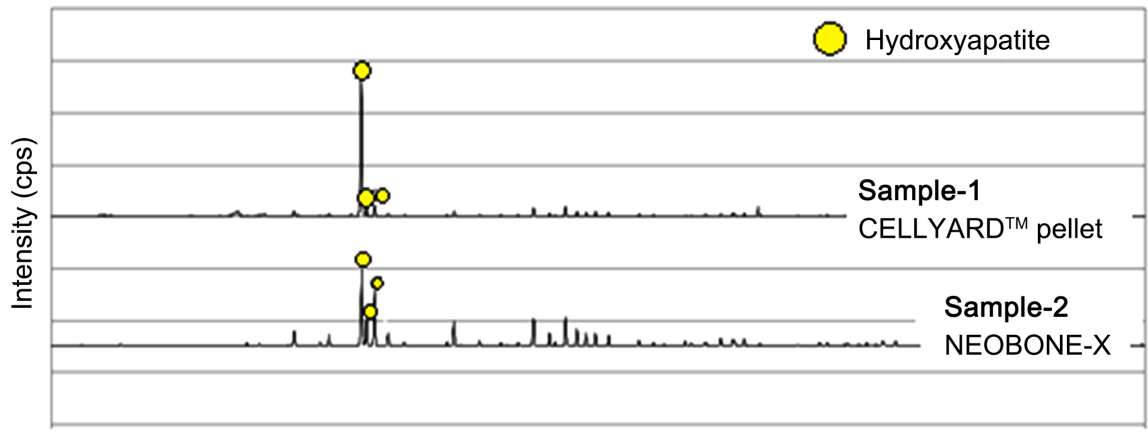

$2 \theta\left(^{\circ}\right)\left(\mathrm{CuKa}_{1}\right)$

(a)

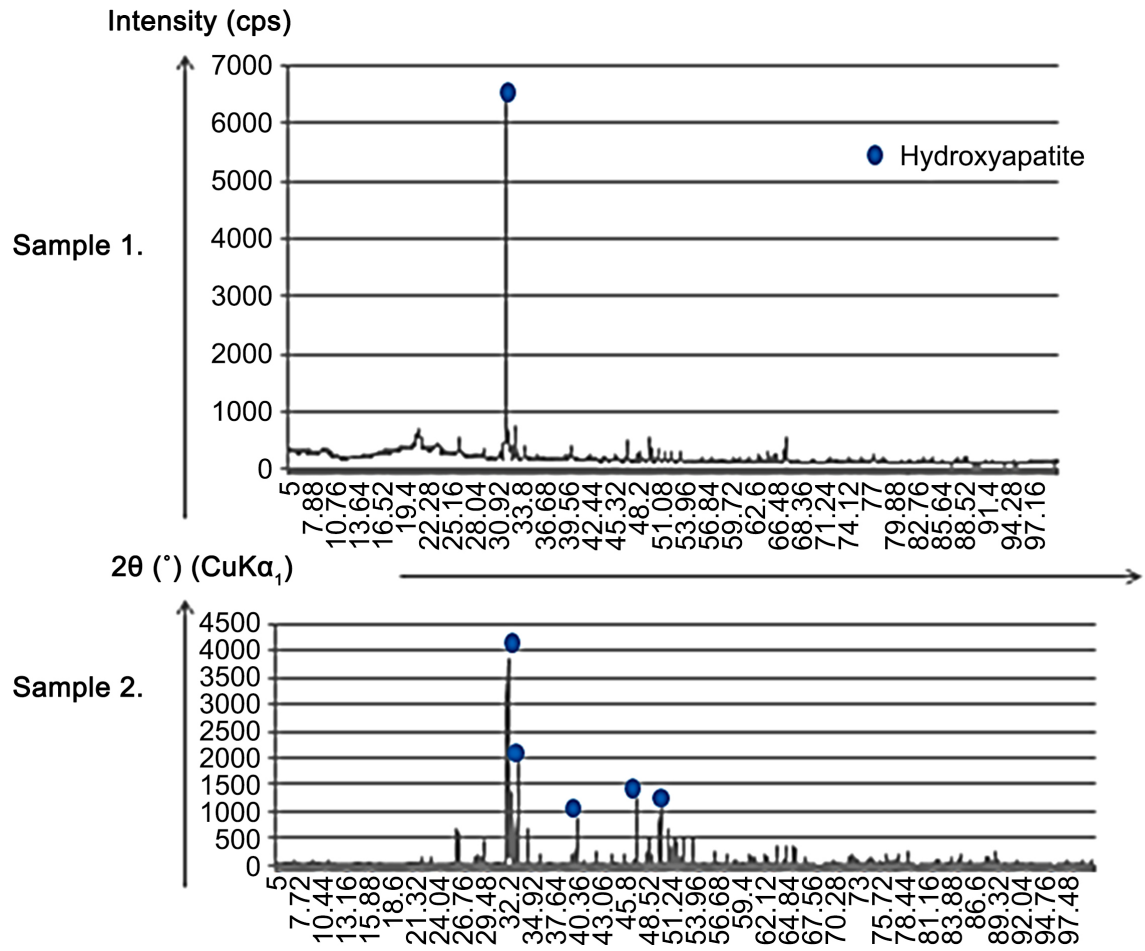

(b)

Figure 4. X-ray diffraction (XRD) of the HA surface before/after soaking in SBF. (a) Before SBF soaking; (b) After SBF soaking for 2 weeks.

These data suggested a series of chemical processes in which the precipitation observed in SEM images were adsorbed and deposited onto the HA surface, and then transformed from calcium phosphate into hydroxyapatite crystalline.

\subsection{Measurement of the Mass of Ca-P Crystallization}

Figure 5 shows the measured sample mass change between before and after SBF soaking, which presents the mass of bone-like precipitation (calcium phosphate crystalline) on HA sample surface. In low-intensity pulsed ultrasound (LIPUS) stimulation group, this precipitate showed significant increases as compared with that in the control group after 1 week and 2 weeks of soaking in SBF ( $p<$ 0.05). 


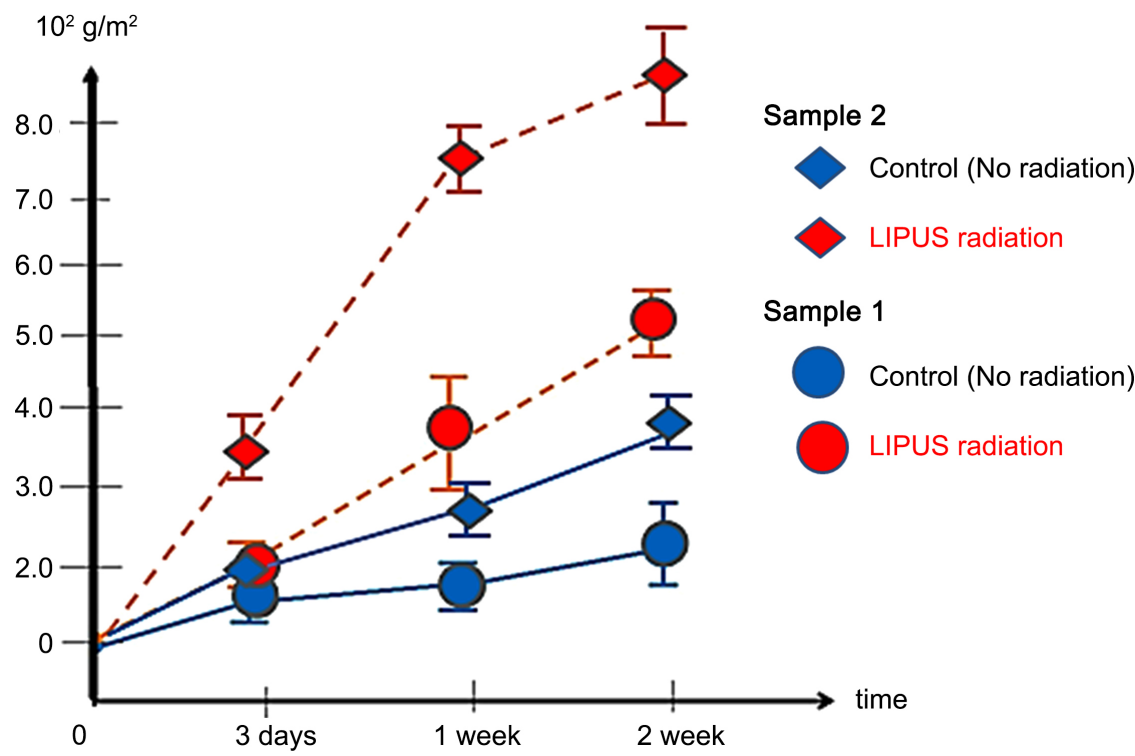

Figure 5. Soaking time dependence of the mass of the precipitation formation on HA surface of specimens (radiation distance: $50 \mathrm{~mm}$ ).

\section{Discussion}

The simulation method using SBF soaking in this study is well known as an in vitro assay for the evaluation of bone-bonding ability of biomaterials, and it has been already used widely for studies on osteoconductivity [7] [14] [15].

According to Kokubo, who developed SBF, the osseointegration of biomaterial was mainly due to mechanisms involving similar steps: firstly calcium phosphate precipitates and grows on the implant material surface as bone-like apatite, then this apatite layer undergoes secondary progression in bonding to the bone tissue grown by biological mechanisms in vivo, and further biological bone modeling surrounding the implant follows. Therefore, successful in vivo bonebonding of bio-active material is dependent on the first apatite formation step on the surface.

LIPUS therapy has been shown to enhance osteogenesis and is widely used as clinical treatment for complicated bone fractures in the field of orthopedic surgery, and it is often speculated that the enhancement of osseointegration of HA implants using LIPUS might be mainly due to bone ingrowth of bone tissue surrounding the implant.

However, as shown by our results from the SBF soaking test, non-biological bone-like apatite formation on the material is also enhanced by LIPUS. This fact indicated that the partial concentration and interatomic potential of $\mathrm{Ca}$ and $\mathrm{PO}_{4}$ ions in SBF can be changed by ultrasound microvibration, which accelerated the nucleation and deposition of ions, resulting in the crystallization of calcium phosphate on the specimen surface. Cavitation by ultrasound waves might also contribute to the formation of the apatite layer by means of the microfracturing of calcium-phosphate and the circulation of new $\mathrm{Ca}$ and $\mathrm{PO}_{4}$ ions of $\mathrm{SBF}$ on the titanium surface. These environmental changes can accelerate further changes in 
the interatomic potential of surrounding atoms, and deteriorate the thermal stability of the calcium-phosphate phase versus $\mathrm{Ca}$ and $\mathrm{PO}_{4}$ ions.

This also supports other clinical reports, and strongly suggests the possibility of the enhancement of osseointegration using LIPUS [16] [17].

In addition, since our data indicated the enhancement mechanism of apatite formation on HA surface by LIPUS can progress through not only the biological pathway but also crystallographic apatite crystallization on the implant surface, the initial stage of osseointegration and osteo-conductivity could be depend on the modification and characterization of the HA surface as a crystallization mechanism.

Both of two kinds of HA sample used in this experiment were high density hydroxyapatite, while, have a little different lattice structure as shown in Figure 5(a). The difference of initial size and amount of apatite crystalline on HA samples in soaking for 3 days in Figure 4 might reflect this difference of microstructure of HA surfaces.

The following Figure 6 shows the SEM images of both HA samples in soaking for one day. The shape of apatite precipitation in both sample surfaces are quadrangular plate-like type, which suggested that this nucleation and epitaxial growth might proceed in Stranski-Krastanov (SK) mode or Volmer-Weber (VW) mode. Considering the hydroxyapatite lattice structure, the epitaxial nucleation and crystal growth of these crystallizations in two samples would form along to the direction of c-axes in hydroxyapatite crystal lattice, however, the amount of them

\section{Spample 1}

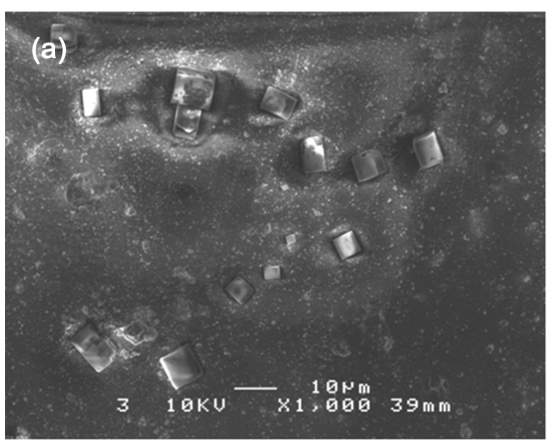

\section{Spample 2}

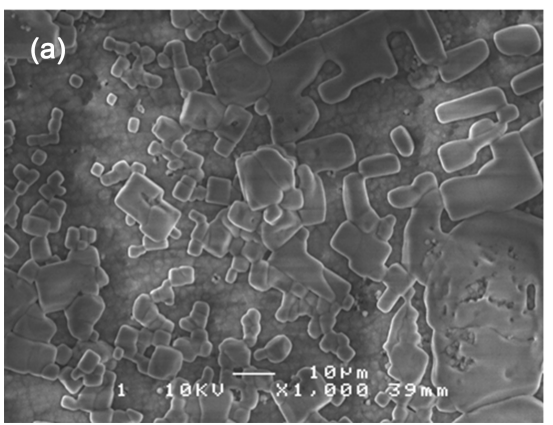

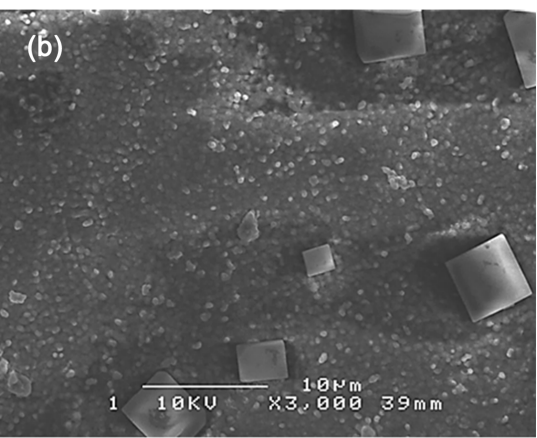

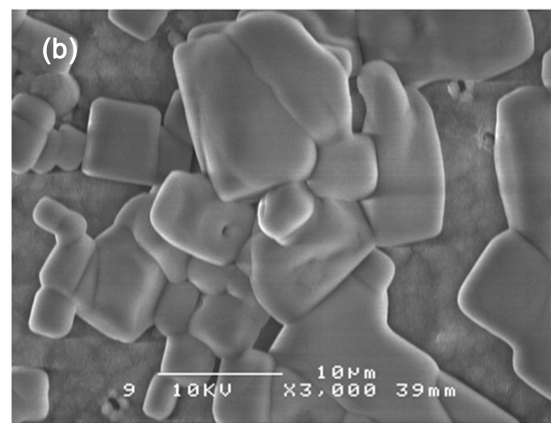

Figure 6. SEM image of the HA implant surfaces in SBF soaking for one day. (a) 1000×; (b) $3000 \times$. 
showed apparent difference. Since atomic arrangement of calcium phosphates spontaneously match with the faced matrix atomic orientation and integrate the structure and finally form the crystallization in nucleation process in SBF, this SEM image make us speculate that Sample-2 surface has more adequate epitaxial interfaces for initial nucleation. X-ray diffraction pattern in Figure 4, which showed that sample-2 revealed more apatite reflection spectra, also indicated the sample-2 is composed of many hydroxyapatite grains with different crystal orientation.

As SEM images already have suggested, LIPUS could accelerate the crystal growth of bone-like apatite after the initial nucleation. For initial epitaxial nucleation, however, the crystallographic characteristics of HA surface should be considered.

This study suggested the therapeutics using LIPUS may be expected as a clinical approach to improving the bone-HA implant bonding, while, the micro-surface structure and crystal orientation of HA surface were also very important for the enhance of apatite formation, osseointegration and osteo-conductivity by LIPUS.

\section{Conclusions}

This study suggested that the clinical application of LIPUS irradiation has great potential for the enhancement of osseointegration or bone attachment of HA implants through the promotion of nucleation and crystallization of bone-like apatite on implant surfaces. Furthermore, the crystallographic characteristics of HA surface should be also considered.

In order to get more detail information regarding clinical application for the enhancement of bone-like apatite formation by the ultrasound wave irradiation, further study concerning the apatite formation on the surface of more different kind of HA microstructure would be necessary.

\section{Conflicts of Interest}

The author declares no conflicts of interest regarding the publication of this paper.

\section{References}

[1] Brånemark, P.I. (1983) Osseointegration and Its Experimental Background. Journal of Prosthetic Dentistry, 50, 399-410. https://doi.org/10.1016/S0022-3913(83)80101-2

[2] Albrektsson, T. and Johansson, C. (2001) Osteoinduction, Osteoconduction and Osseointegration. European Spine Journal, 10, S96-S101. https://doi.org/10.1007/s005860100282

[3] Thomas, K.A., Kay, J.F., Cook, S.D. and Jarcho, M. (1987) The Effect of Surface Macrotexture and Hydroxyapatite Coating on the Mechanical Strength and Histologic Profiles of Titanium Implant Materials. Journal of Biomedical Materials Research, 21, 1395-1414. https://doi.org/10.1002/jbm.820211205

[4] De Andrade, M.D., Sader, M.S., Filgueiras, M.R.T. and Ogasawara, T. (2000) Microstructure of Ceramic Coating on Titanium Surface as a Result of Hydrothermal Treatment. Journal of Materials Science: Materials in Medicine, 11, 751-755. 
https://doi.org/10.1023/A:1008984030540

[5] Søballe, K. (1993) Hydroxyapatite Ceramic Coating for Bone Implant FixationMechanical and Histological Studies in Dogs. Acta Orthopaedica Scandinavica, 64, 1-58. https://doi.org/10.3109/17453679309155636

[6] Smeets, R., Stadlinger, B., Schwarz, F., Beck-Broichsitter, B., Jung, O., Precht, C., Kloss, F., Alexander Gröbe, A., Heiland, M. and Ebker, T. (2016) Impact of Dental Implant Surface Modifications on Osseointegration. BioMed Research International, 2016, Article ID: 6285620. https://doi.org/10.1155/2016/6285620

[7] Yamaguchi, S., Akeda, K., Murata, K., Takegami, N., Goto, M. and Kokubo, T. (2015) Chemical and Heat Treatments for Inducing Bone-Bonding Ability of Ti-6Al-4V Pedicle Screw. Key Engineering Materials, 631, 225-230.

https://doi.org/10.4028/www.scientific.net/KEM.631.225

[8] Kobayashi, M. (2017) Study on Enhancement of Osseointegration of the Bio-Active Titanium Implant by Low Intensive Ultrasound Wave. (Part 1: Simulated Body Fluid Soaking Test). International Journal of Biomedical Engineering and Science, 4, 1-10

[9] Kobayashi, M., Shiraishi, M. and Kinoshita, M (2018) Study on Enhancement of Osseointegration of the Bio-Active Titanium Implant by Low Intensive Ultrasound Wave. (Part 2: In Vivo Experiment). International Journal of Biomedical Engineering and Science, 5, 1-10 https://doi.org/10.5121/ijbes.2018.5101

[10] Kobayashi, M. (2020) Enhancement of Osseointegration of Hydroxyapatite Implants by Low-Intensity Ultrasound Irradiation. Journal of Osseointegration, 12, 1.

[11] Kokubo, T. and Takamada, H. (2006) How Useful Is SBF in Predicting in Vivo Bone Bioactivity? Biomaterials, 27, 2907-2915. https://doi.org/10.1016/j.biomaterials.2006.01.017

[12] Kokubo, T., Miyaji, F., Kim, H.M. and Nakamura, T. (1996) Spontaneous Formation of Bonelike Apatite Layer on Chemically Treated Titanium Metals. Journal of the American Ceramic Society, 79, 1127-1129. https://doi.org/10.1111/j.1151-2916.1996.tb08561.x

[13] Kokubo, T. and Yamaguchi, S. (2010) Novel Bioactive Titanate Layers Formed on Ti Metal and Its Alloy by Chemical Treatments. Materials, 3, 48-63. https://doi.org/10.3390/ma3010048

[14] Mukai, H. and Kobayashi, M. (2011) The Effect of Low-Intensive Pulsed Ultrasound Waving on Hydroxyapatite in Simulated Body Fluid. 2011 IEEE 11th International Conference on Bioinformatics and Bioengineering, Taichung, 24-26 October 2011, 125-128. https://doi.org/10.1109/BIBE.2011.27

[15] Zhao, W., Michalik, D., Ferguson, S., Hofstetter, W., Lemaître, J., von Rechenberg, B. and Bowen, P. (2019) Rapid Evaluation of Bioactive Ti-Based Surfaces Using an in Vitro Titration Method. Nature Communications, 10, Article No. 2062. https://doi.org/10.1038/s41467-019-09673-1

[16] Abdulhameed, E.A., Enezei, H.H., Omar, M., Komori, A., Sugita, Y., Hegazy, F.A., Ari, S., Maeda, H. and Alam, M.K. (2017) The Effect of Low Intensity Pulsed Ultrasound Therapy on Osseointegration and Marginal Bone Loss Around Dental Implants. Journal of Hard Tissue Biology, 26, 323-330.

https://doi.org/10.2485/jhtb.26.323

[17] Khomich, I., Rubnikovich, S. and Khomich, S. (2015) Enhancing Osseointegration and New Bone Formation around Dental Implants Using Low Intensity Pulsed Ultrasound. International Journal of Oral \& Maxillofacial Surgery, 44, E243. https://doi.org/10.1016/j.ijom.2015.08.178 